\title{
A Location Context Awareness Personalized Mobile Service Prediction Model and it's Algorithm: Design and Implementation
}

\author{
Minjun Xin, Liyuan Zhou, Han Cao and Zhihua Niu \\ School of Computer Engineering and Science, Shanghai University, \\ Shanghai 200444, China \\ xinmj@shu.edu.cn,lyozhou@139.com,shu_caohan@163.com, \\ zhniu@staff.shu.edu.cn
}

\begin{abstract}
With the expansion of mobile Internet, location-based services have become a hot spot in Internet industry. To improve the accuracy and efficiency of the location-based services discovery, researchers in web services recommendation area are still busy looking for method. In this paper, it proposes a LCAMSP model (Location Context Awareness Mobile Service Prediction, LCAMSP) under mobile Internet environment, aims to meet the exact personal requirements of users' current location and preference. Then, the similar users' grouping is also a important thread to predict the continuous movement for mobile users. Because each user has own preference, a dynamic calculation function for the weight of each attribute is discussed in this paper. Finally, taking the hotel reservation service as an example, a verification algorithm will be applied to measure the performance of LCAMSP method. With the premise of increasing limited time, the accuracy of LCAMSP algorithm is significantly improved under the mobile service environment.
\end{abstract}

Keywords: Location-based services, mobile Internet, services discovery, location context

\section{Introduction}

In recent years, with the popularity of smart phone, mobile terminal is the most convenient access to discover the Internet resources [1]. People can immediately get desired info through the query on mobile phone. The technology of intelligent devices and mobile communication also provide the response to different kinds of services for location-based services (LBS), which pushes surrounding commercial information to different users.

Due to a great variety of services generated on the Internet everyday, information overload and information lost are considered to be the bottle-neck for service providers in recent years. The most important characteristic of the mobile client is the real-time location context [2], which is a significant difference from the traditional mode of electronic commerce. How to get the users' location context in time and analyze them on server has been the research hotspot for mobile services.

The existed algorithms proposed by the previous research institutes [3] on web-based service recommendation are all designed on the basis of improved recommendation algorithms for web services. However, as an important element for location-based service recommendation, the location context hasn't paid enough attention. The existing research makes the location context as one attribute for different users, which will reduce the importance of location context. It is also unilateral for the algorithms which take the single filter based on location context. How to meet the special needs under 
mobile Internet environment is a comprehensive computational process which includes a lot of related elements. Therefore, it is necessary to design an improved strategy which can reasonably set the weight of users' preference ${ }^{[4]}$ and location context.

In this paper, it proposes an improved framework of mobile service discovery based on the location context. Then it combines the contents of location context with users' preference. The method of ontology modeling [5] is used to describe the involved elements in the services prediction. The weight matric can be calculated in the predication model which has been customize according to different attributes of the services for each user. After the personalization filtering, the user's current location context will be compared with the historic records to acquire a optimal solution. In brief, the recommended services results will be discovered on the basis of computing the similarity of mobile service and users' preference according to their location context.

Section 2 mainly focuses on the weakness of current services discovery technology. Then, an improved service discovery framework is proposed in Section 3, the process of the services prediction and the implementation methods are described in details. In Section 4, the LCAMSP algorithm is proposed and some samples are presented to show the code flow. In Section 5, the experiments are designed to test the accuracy and efficiency of LCAMSP algorithm. Finally, the conclusion and outlook for our research work is discussed in Section 6. In brief, on the basis of computing the similarity of mobile service and user's preference according to their location context, LCAMSP could predict and recommend possible and available services results to different users.

\section{Research Status}

At present, the methods of web service discovery mainly focus on the grammatical level and semantic level [6]. For the former, it mainly compares the information of service' ID, name, functions with keywords, which is widely used in famous companies. While for the latter, it uses a certain logical reasoning mechanism based on domain ontology or large vocabulary to search the most suitable service for different users [7].

The application of context discovery is first proposed in 2010 by Google, which can push the information to the user automatically. Mechanism behind it is using contextaware service computing to personalized recommendation results. In addition, a service discovery method [8] based on context clustering provides a new contextualized user preference model [9] for personal mobile service recommendation.

In order to qualify the personalized recommendation model with the ability of context sensitive, the current trend is to make the information more contextual. It represents context awareness attributes should be put into the procedure of the service discovery strategy. After that, the recommend results might be considered as the most suitable service to the user with his location context. Currently, Chinese scholars mainly concerned about the research on personalized recommendation without context. Xu Hailing etc., [10] proposes a formal and non-formal definitions for recommended question and classified the mainstream algorithms. Cai Zhihong [11] discusses five services recommended modes based on the view of user and introduces the related research topics separately. Wu Lihua [12] classifies the user modeling techniques for personalized recommendation systems, summarizes and discusses the four topics of the data gaining in user modeling, the expression of user model, learning and updating.

For the current status of research and application, the traditional models mainly focus on the semantic extension in services discovery. What's more, the relationship between users' historic information and the current one is neglected in traditional model. In 
addition to the attributes matching between achieved services and requirements, users' needs can be predicted through the analysis on the records of similar users. For this reason, the researchers pay more and more attention on collaborative filtering.

Most of the current recommendation systems mainly focus on how to provide users with the most suitable service that fit their needs while neglecting the fact that dynamic context will have a great impact on final output for service users. The vague user preferences will lead to the inaccurate result to user as well. Traditional strategy mostly discovers the services from semantic level. Current study such as literature [13-14] focuses on the improvement of the semantic matching. The huge information about the service records of the similar user is a valuable resource. Through the resource, we can accurately imitate the current user's requirement to get the mobile service result.

With the premise of summarizing the advantage and disadvantage of the current web service discovery methods, a personalized location-based services prediction framework based on location context is put forward in this paper. It is effective to solve the problem on how to match location context and calculate user preference dynamically. First of all, the users' preference and location context are described by semantic ontology. In addition, this paper will also cover the mathematical formulas used in the algorithms. Then, the service filters for users' preference and location context are realized in the form of code separately, and a case will show the detail procedure of the workflow according to the process in the framework. Finally, the experiments for the service prediction model prove that the accuracy has been greatly improved.

\section{A Location Context Awareness Mobile Services Prediction Model}

In this paper, the character of personalized recommendation and location context awareness is significantly different from the traditional services discovery strategies. In this section, the processes of the improved framework will be discussed in detail.

\subsection{Services Prediction Framework}

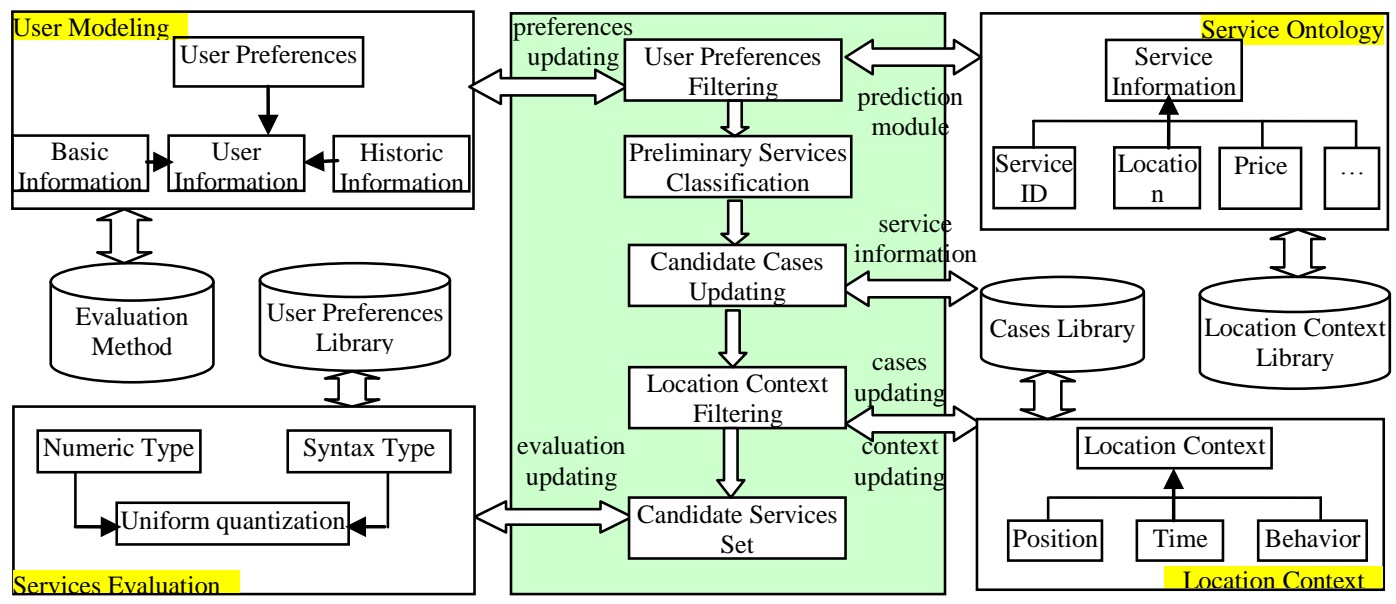

Figure 1. A Framework of Context Awareness Mobile Services Prediction

First, the request is uploaded to the server through terminal in the services prediction model. Second, the server filters the achieved services according to the request. If there is a similar user who has a same request, the model will return the existing record to the user. 
Thus, the efficiency will be improved. Otherwise, as is shown in Figure 1, the matching degree between the candidate service and request about each attribute will be calculated through the model. By means of combining the matching degree and preference weight, we can get the satisfaction degree. Finally, the server collects the current context information to compare with the historic data. The final filtering will recommend the optimal services to users.

The function of the each part can be described as follows:

1) User preferences filter: Through the data mining to calculate the historic user information, the prediction model determines the users' preference to different categories of service. With the help of user preference, it can get a more suitable service set from the massive data as the objects in the subsequent filtering process.

2) Preliminary services classification: The results from preference filtering will be used in the service classification. The main steps in this process include the analysis of the Chinese word, termination word filtering, the pretreatment of service description data, the description documents of training service and service classifier building.

3) Candidate cases update: Check whether there exist the same instances in the historic record according to the preference and request. If there are some similar records in the database, the server will directly return the historic records to the user and the query efficiency will be improved according to the candidate cases.

4) Location context filter: For the mobile users with uncertainty position, each of them has various context-aware attributes under mobile Internet environment, such as weather, time, season, location and so on. Thus, we set up the context library and save the historic context for service recommendation. Then, the secondary filtering can be finished by the similarity of the location context.

At the end of the prediction process, the most suitable results of service discovery will be returned. As discussed in Figure 1, the model collects the evaluation for user satisfaction rating and stores it to the database.

Since there are many factors under the mobile environment, an active mechanism is proposed in the process of services prediction, which can describe the information of the objects clearly. The ontology modeling technology can solve this problem effectively.

\subsection{Location Context Awareness Ontology Modeling}

The user information always includes ID, contact way, gender. The function of these attributes is to distinguish users, while the historic information is the previous access records and evaluation for the recommendation system.

Since everyone has different kind of preference, different users can choose different kinds of services. Taking the travel services as an example, for the undergraduate students, they generally choose the economy hotels, while business people may prefer business-type hotels to meet their needs. For different kind of users, the concept of ontology modeling should be used as description tool for the next step of preferences matching.

\section{Definition 1: User Ontology}

$$
\text { User }=\left(U_{I D}, U_{\text {Age }}, U_{\text {Career }}, U_{\text {Price }}\right)
$$


User ontology modeling involves ID, age, occupation and acceptable price range. The acceptable price range will be mapped to the real number in this paper.

Service information is the attribute information of the candidate service which includes ID, price, location attribute and so on. The data for the experiment in this paper is provided by the "elong.com", a company provides detail information of hotel, flight to both domestic or abroad traveler. The attributes include ID, name, price, star, facility and location.

\section{Definition 2: Service Ontology}

$$
\text { Service }=\left(S_{I D}, S_{\text {Name }}, S_{\text {type }}, S_{\text {price }}, S_{\text {facility }}, S_{\text {Position }}, S_{\text {Commen }}\right)
$$

The value of the attributes can be divided into two type, the numeric one and discrete one. The former type includes price, distance and location. The latter includes Wifi, broadband, parking lot, gymnasium and other hotel facilities. Hotel types include business, express. The attributes in the service ontology are the parameters to compare with the achieved services and the requirement of user.

\section{Definition 3: Context Ontology}

$$
\text { Location }=\left(\boldsymbol{L}_{I D}, \boldsymbol{L}_{\text {Position }}, \boldsymbol{L}_{\text {Time }}, \boldsymbol{L}_{\text {Weather }}, \boldsymbol{L}_{\text {season }}\right)
$$

Context ontology includes the geographic location of user which can be used to search for the achieved service in the certain range. The other attributes like time, reason, and weather which will impact the needs of different users. We can harness these dynamic context attributes to make a quick reaction for the personal requirement of a users under the mobile Internet environment.

It is different from the traditional methods which are based on grammar. The prediction method proposed in this paper is based on semantic under mobile Internet environment. The restriction of the grammar attributes is broken by the ontology model. User, service and location are the most three important factors in the implementation of the prediction method proposed in this paper. The attributes discussed in the above definitions above are refined form the feature of each object. By means of combining them, users' preference for different kinds of services under a certain location could be clarified clearly. After the prediction model completes the calculation for the related objects, the final output service can meet the user need.

\subsection{The Process of Services Matching}

User has different needs for different kinds of service attributes, the attribute values are divided into 2 types, numeric and discrete. In this paper, the first filter of the recommendation strategy focuses on matching user preference, and then it triggers the second filter based on the location context under mobile environment.

For the different attributes and value types, we need to design a method to describe the matching degree between service and the user's preference.

\section{Definition 4: Service Matching Degree}

Calculating the similarity between the value of all the attributes of the services and the request for the different attributes of a user. The sum is the value of services match degree.

In this paper, we define the value of the $l_{t h}$ attribute for the $i_{t h}$ service as $P_{i l} \cdot P_{i l}$ is a numeric attribute. $P_{i k}$ is the value for a discrete attribute(k>1) of the $\boldsymbol{i}_{t h}$ service. 
The acceptable range of value for user is $\left[c_{i l}, d_{i l}\right]$, the most suitable value is $\boldsymbol{e}_{i l} \cdot S_{i k}$ is

the user request for the $k_{t h}$ discrete attribute of the $i_{t h}$ service. We can use the following formulas on behalf of the service matching degree.

Formula(1) is the definition for the type of numeric attributes:

$$
X_{i l}=\left\{\begin{array}{l}
\left.\alpha P_{i l}<c_{i l} \text { 或 } P_{i 1}>d_{i l}\right) \\
\frac{P_{i l}-c_{i l}}{e_{i l}-c_{i l}}\left(c_{i} \leq P_{i l}<e_{i l}\right) \\
\frac{d_{i 1}-P_{i l}}{d_{i l}-e_{i l}}\left(e_{i l} \leq P_{i l}<d_{i}\right) \\
1\left(P_{i l}=e_{i l}\right)
\end{array}\right.
$$

Formula(2) is the definition for the type of discrete attributes:

$$
X_{i k}=\frac{\operatorname{Count}\left(S_{i k} \cap P_{i k}\right)}{\operatorname{Count}\left(S_{i k}\right)}
$$

The Formula(1) and the Formula(2) are used to calculate the result of service matching degree, but the value only reflects the matching degree of each attribute .In the next section, we will explain how to get the overall satisfaction for the mobile service by the matching degree and the weight.

Now, taking the user in Table 1 as example, we assume that one user wants to search a hotel, the request detail is shown in the Table 1 and Table 2.

Table 1. The User Request about the Hotel

\begin{tabular}{lccc}
\hline Type & Location & Price( $¥)$ & Facility \\
\hline Business & L1 & 150 & Wifi, gymnasium \\
\hline
\end{tabular}

From Table 1, we can know that the user wants to get a hotel whose type is business, location is L3, price is $¥ 180$, and the facilities of the hotel should contain Wifi and gymnasium. According to the data mining result from the search engine, the range of the price that the user can accept is $¥ 80 \sim ¥ 200$.

One record of hotel information can be got from Table 2. Take the attributes of price and facility as example.

Table 2. The Hotel Information

\begin{tabular}{lccccc}
\hline ID & Name & Type & Location & Price( $¥)$ & Facility \\
\hline 0001 & RuJia & economy & L1 & 160 & Wifi \\
\hline
\end{tabular}

For the attribute of price, the objects in the formula can be showed as $P=160, c=80, d=200, e=150$. According to the Formula(1), the matching degree is $X=\frac{d-P}{d-e}=\frac{200-160}{200-150}=0.8$. 
For the attribute of facility, the objects in the formula can be showed as $S=($ wifi, gymnasium $), P=($ wifi $)$. According to the Formula(2), the matching degree is $X=\frac{\operatorname{Count}(S \cap P)}{S}=\frac{(\text { wifi, gymnasium }) \cap(\text { wifi })}{\text { (wifi, gymnasium })}=0.5$.

These two calculations are designed for the numeric attribute "price" and the discrete attribute "facility". Both the two type attributes can be calculated in the two formulas discussed above.

In all, a services prediction framework based on location context is proposed in this paper, and it describes the functions of each module in the framework and shows the implementation process in detail. Furthermore, the objects involved in the service discovery are introduced in the form of ontology. Finally, the definition of service matching degree indicates the standard during the service matching under mobile Internet environment.

\section{The Implementation of the LCAMSP Algorithm}

In order to improve the accuracy of the mobile service discovery, we emphasize on adding the dynamic preference and location context into the mobile service discovery model in this paper. The result of the LAMSP model can adjust immediately when the mobile Internet environment for the user is changed. In this section, the key code and the core principle of the LCAMSP model will be introduced in detail.

\subsection{Calculation for the Preference Weights}

The standardized calculation for the numeric and discrete attributes has been already mentioned previously. Because each user has the personalized preference attribute, this section will focus on how to calculate the weight of different attributes.

The range of numeric attribute is inversely proportional to the user preference. If the range of the numeric value is larger, it shows the lower degree of requirement for this attribute. On the contrary, for the discrete attribute, the more items user has selected in the collection, the more the user care these attributes of the selected items.

The weight for the $a_{t h}$ numeric attribute of the $i_{t h}$ service can be described as follow:

$$
\mu_{a}=\frac{1-\frac{d_{i a}-c_{i a}}{\max \left(P_{i a}\right)-\min \left(P_{i a}\right)}}{\sum_{a=1}^{l}\left(1-\frac{d_{i a}-c_{i a}}{\max \left(P_{i a}\right)-\min \left(P_{i a}\right)}\right)+\sum_{a=l+1}^{k}\left(\frac{\operatorname{Count}\left(S_{i a}\right)}{\operatorname{Count}\left(\cup P_{*_{a}}\right)}\right)}
$$

$\cup P_{* a}$ is the union of the $a_{t h}$ discrete attributes among all the achieved services. The discrete attributes can be described as:

$$
\boldsymbol{\mu}_{a}=\frac{\sum_{a=l+1}^{k}\left(\frac{\operatorname{Count}\left(\boldsymbol{S}_{i a}\right)}{\operatorname{Count}\left(\cup \boldsymbol{P}_{*_{a}}\right)}\right)}{\sum_{a=1}^{l}\left(1-\frac{d_{a}-\boldsymbol{C}_{a}}{\max \left(\boldsymbol{P}_{i a}\right)-\min \left(\boldsymbol{P}_{i a}\right)}\right)+\sum_{a=l+1}^{k}\left(\frac{\operatorname{Count}\left(\boldsymbol{S}_{i a}\right)}{\operatorname{Count}\left(\cup \boldsymbol{P}_{*_{a}}\right)}\right)}
$$

Due to the limitation of the paper, we select three attributes named "price", "comment" and "facility" separately as the sample to get their weights separately. There are 6 pieces of data records in the Table 3 we can get. 


\begin{tabular}{|c|c|c|c|}
\hline HOTEL ID & Price( $¥$ ) & Facility & Comment \\
\hline 0001 & 160 & Wifi & 蚁坟 \\
\hline 0002 & 60 & l & $\star \star \star *$ \\
\hline 0003 & 200 & Wifi, broadband & 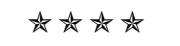 \\
\hline 0004 & 180 & Wifi,parking lot & 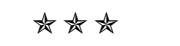 \\
\hline 0005 & 680 & $\begin{array}{l}\text { Wifi,parking lot } \\
\text { broadband, } \\
\text { gymnasium }\end{array}$ & 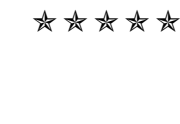 \\
\hline 0006 & 100 & Wifi & 虾 \\
\hline
\end{tabular}

According to Table 3 , the range of price for all available resources is $¥ 60 \sim ¥ 680$, the range of comment is $2 \sim 5$, the available facilities are defined as (Wifi, parking lot, broadband, gymnasium). Assuming that the acceptable price range for the user is set to $¥ 80 \sim ¥ 200$, the comment is $3 \sim 5$, so the user's requirement for the facilities of the hotel is deduced to the set of (Wifi, gymnasium).

Firstly, the "price", "comment", "facility" attributes can be calculated as follow:

$$
\begin{aligned}
& \omega_{\text {price }}=1-\frac{d_{i a}-c_{i a}}{\max \left(P_{i a}\right)-\min \left(P_{i a}\right)}=1-\frac{200-80}{680-60}=0.8 \\
& \omega_{\text {comment }}=1-\frac{d_{i a}-c_{i a}}{\max \left(P_{i a}\right)-\min \left(P_{i a}\right)}=1-\frac{5-3}{5-2}=0.3 \\
& \omega_{\text {facility }}=\frac{\operatorname{Count}\left(S_{i a}\right)}{\operatorname{Count}\left(\cup P_{* a}\right)}=\frac{(\text { wifi, gymnasium })}{(\text { wifi, parking_lot, broadband, gymnasium })}=0.5
\end{aligned}
$$

Secondly, the results of the preference weights can be calculated as follows:

$$
\begin{aligned}
& \mu_{\text {price }}=\frac{\omega_{\text {price }}}{\left(\omega_{\text {price }}+\omega_{\text {commen }}\right)+\omega_{\text {facility }}}=\frac{0.8}{(0.8+0.3)+0.5}=0.5 \\
& \mu_{\text {comment }}=\frac{\omega_{\text {comment }}}{\left(\omega_{\text {price }}+\omega_{\text {commen }}\right)+\omega_{\text {facility }}}=\frac{0.3}{(0.8+0.3)+0.5}=0.2 \\
& \mu_{\text {facility }}=\frac{0.5}{\left(\omega_{\text {price }}+\omega_{\text {comment }}\right)+\omega_{\text {facility }}}=\frac{0.5}{(0.8+0.3)+0.5}=0.3
\end{aligned}
$$

Through formulas metioned above, we can dynamically calculate the weight of the different user preference. Compared with the traditional methods which define the preference weight manually, the method in this paper can reflect the user preference more actual under the mobile services environment.

\subsection{Calculation for the User Satisfaction}

After calculating of service match degree and preference weights, the overall satisfaction $\omega_{i}$ can be defined as Formula(6) 


$$
\omega_{i}=x_{i 1} \bullet \mu_{i 1}+x_{i 2} \bullet \mu_{i 2}+x_{i 2} \bullet \mu_{i 2}+\ldots \ldots+x_{i k} \bullet \mu_{i k}
$$

Service matching degree only shows the user satisfaction for a single attributes, it cannot actually reflects whether the services meet the user's requirements. To find a method which can reflect the overall satisfaction for the services, we should combine service matching degree and preference weights together. The list of the result will be arranged in descending order according to the overall satisfaction. $\omega_{i}$ is the degree measuring how the service meet the user's need.

Algorithm.1: Calculation of overall user satisfaction

Input: request for service from user

Output: the service list

Request(User.need);

//the request from user

For $(\mathrm{i}=0 ; \mathrm{i}<1 ; \mathrm{i}++)\{$

//make the preference of numeric attributes have the same standard

$\mu_{i=\text { numWeightCaculate(); }\}}$

//initialize the user preferences for each attribute $\mu$

For $(\mathrm{i}=1 ; \mathrm{i}<\mathrm{k} ; \mathrm{i}++)\{$

$/ /$ make the preference of discrete attributes have the same standard

$\boldsymbol{\mu}_{i=\text { discreteWeightCaculate(); }}$

Do\{

//initialize the user satisfication for each attribute $\chi$

tempServices=hasNextServices();

//retrieval the candidate services

While(tempServices $)\{$

For $(\mathrm{i}=0 ; \mathrm{i}<1 ; \mathrm{i}++)\{$

//calculate the preference degree for numeric attributes

$\chi_{i=\text { numSatisfactionCaculate }() ;\}}$

For $(\mathrm{i}=1 ; \mathrm{i}<\mathrm{k} ; \mathrm{i}++)\{\quad$ //calculate the preference degree for discrete attributes

$\chi_{i=\text { discreteSatisfactionCaculate }() ;\}}$

$\omega_{\text {=weightSatis }} \mu, \omega$ );

//calculate the comprehensive satisfaction with weights

tempServices=hasNextServices(); $\}\}$

Return ServiceList():

//return the services set which reach the threshold

In Algorithm.1, it calculates the service matching degree and the user preference weights, then it calls the function WeightSatis() and get the results in descending order. On the basis of combining these two metrics, a good effect has been achieved on the results.

\subsection{The Similarity Calculation of Location Context}

After comparing the information of the user location context with the historical records in database, the system will filter the results from Algorithm1 and get the optimal results according to the similarity of location context. 
In algorithm.2, we compare current location context with the historic records, and get the most similar service set.

Algorithm.2: Calculation of the Similarity of Location Context

Input: the current location context, the historical location context of the services set

Output: the final prediction result

Begin

Get(User_Location); //get the information of the current location context //Initialize the object SimilarLocation, make it point to the most similar historic location Do\{ tempLocation=hasNextLocation ()$; \quad$ //retrieval the historic location context library While(tempLocation ) \{

Degree_SimilarCaculate(tempLocation) //calculate the similarity If(SimilarLoaction.value<tempLocation.value $)\{\quad / / m a k e$ SimilarLoaction // point to the historic location context with the highest similarity degree SimilarLoaction $=$ tempLocation;

\}\}\}

Return GetSimilarServices(SimilarLoaction); //return the final prediction result

Through Algorithm.2, we realize the function of similarity filtering of location context. On the basis of the first filter, the prediction model takes the location context into account so that the accuracy of the final result will be improved.

These two algorithms are the key of the service prediction platform. In Algorthm.1, the system calculates the matching degree between the request and all the available resources, then it calculates the preference weights for each attributes, the time complexity is $O\left(n^{2}\right)$.

In Algorithm2, the algorithm matches the current location context with each historic record and return the most similar ones, its time complexity is $O(n)$.

Algorithms above improve the accuracy of services prediction. In the next chapter, a case will be gone through in order to verify the performance of LCAMSP.

\section{Experiment of the LCAMSP Algorithm}

The experiments are designed to test the effectiveness of the LCAMSP algorithm. The time spending and the accuracy are tested in this section to prove the improved effect and feasibility.

\subsection{Calculation Process}

The example in this section shows the procedures of the LCAMSP algorithm. The client runs on the OS environment of android. The server is set up with the technology of ".Net" supported by Microsoft. There are about 500 records in SQL Server 2008 
including all types of hotels. We assume that a user who is in loaction 1 wants to get some suitable hotel information.

Step1: The user sends the request

Step2: The server collects the user location context in Table 4

Table 4. The Current Location Context of the User

\begin{tabular}{lcccc}
\hline & Location & Time & Weather & Season \\
\hline $\mathbf{F}$ & L2 & night & rainy & winter \\
$\mathbf{N}$ & L1 & night & fine & winter \\
\hline
\end{tabular}

Step3: The server compares the current data with the historic record, if there does not exist any same information of request and location context, we should jump to step4, otherwise we should jump to step 10.

Step4: Then, the server gets the following information shown in Table 5 through mining data from historic consumer records.

Table 5. User Preference

\begin{tabular}{|c|c|c|c|}
\hline Attribute & Maximum & Optimal & Minimum \\
\hline Location & $1000 \mathrm{~m}$ & $500 \mathrm{~m}$ & 0 \\
\hline Price( $¥)$ & 200 & 150 & 80 \\
\hline Comment & \multirow{2}{*}{\multicolumn{3}{|c|}{$\begin{array}{l}\text { Wifi, parking lot } \\
\text { Wh }\end{array}$}} \\
\hline Facility & & & \\
\hline
\end{tabular}

Step5: According to the historic data, the server calculates the preference weights $\mu_{\text {location }}, \mu_{\text {price }}, \mu_{\text {estimate }}, \mu_{\text {faclity }}$.

Step6: Through making the service classification according to the level of consumption, the spending time for services searching can be reduced.

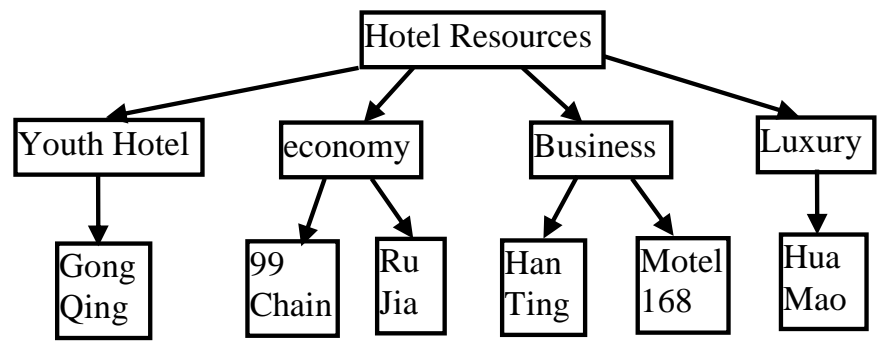

Figure 2. Resources Classification

Step7: According to the requirement information, the server calculates the service match degree $\omega_{\text {Loaction }}, \omega_{\text {price }}, \omega_{\text {estimate }}, \omega_{\text {faclity }}$

Step8: After substituting the parameters into formula for the overall user satisfaction, we can get the result in Table.6. 
Table 6. The Service Information after the First Filter

\begin{tabular}{|c|c|c|c|c|c|c|}
\hline ID & Name & Type & Location & $\operatorname{Price}(Y)$ & Facility & Comment \\
\hline 0001 & RuJia & economy & L1 & 160 & Wifi & 办光光 \\
\hline 0002 & GongQing & youth hotel & L2 & 60 & l & $\star \star \star x$ \\
\hline 0003 & HanTing & business & L3 & 200 & Wifi, Broadband & 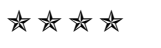 \\
\hline 0004 & Motel168 & business & L1 & 180 & Wifi,parking lot & 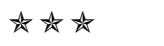 \\
\hline 0005 & HuaMao & luxury & L2 & 680 & $\begin{array}{l}\text { Wifi,parking lot } \\
\text { Broadband, } \\
\text { gymnasium }\end{array}$ & 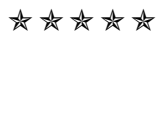 \\
\hline 0006 & 99 Chain & economy & L1 & 100 & Wifi & 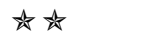 \\
\hline
\end{tabular}

Step9: Compare the current location context with the service in Table. 6 in formula, we can calculate the similarity of location context.

Step10: The server sends the final filtering result to the client, then collects the comment on the services and saves it into database.

\subsection{Time Performance Evaluation}

In order to verify the superiority of the algorithm on the aspect of time, the five experiments with the different number of services will be executed. The number of the test data is $100,300,500,700$ and 900 .

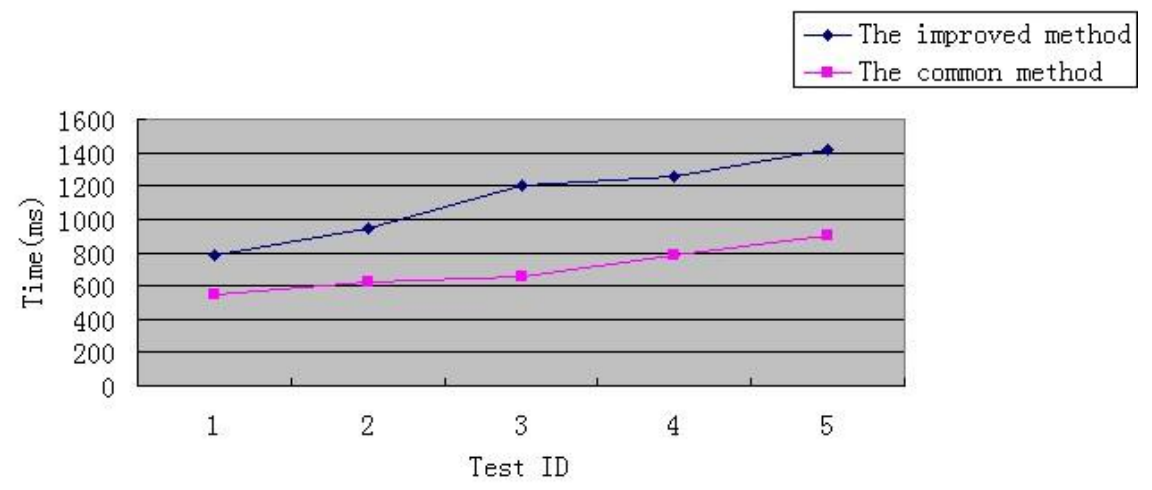

\section{Figure 3. The Time Spending Between Improved Method and Common Method}

The conclusion of the Figure 3 shows the time spending is increased with the increase of test data in the range of millisecond. It shows that the increase of the time is acceptable for user.

\subsection{Results Accuracy Evaluation}

The main purpose of the test is designed to verify the improved method is more accurate than the traditional one. We test 10 random users in different location context and offer each of them 15 services which can meet the request. Each user will subjectively choose 5 services which can be most suitable in his current context. All the location context of the user and the 5 services which they have chosen will be recorded. The 5 chosen services for each user constitute set A. Suppose the user randomly choose 5 services from the 15 services and constitute set B. After above steps, we can input the 
location context of the each user and the 15 services into the improved system, the system handle these data and output the 5 services as set C. So the accuracy of the two way can be expressed as:

$$
\operatorname{Accuracy}(1)=\frac{A \cap B}{B}, \operatorname{Accuracy}(2)=\frac{A \cap C}{C} .
$$

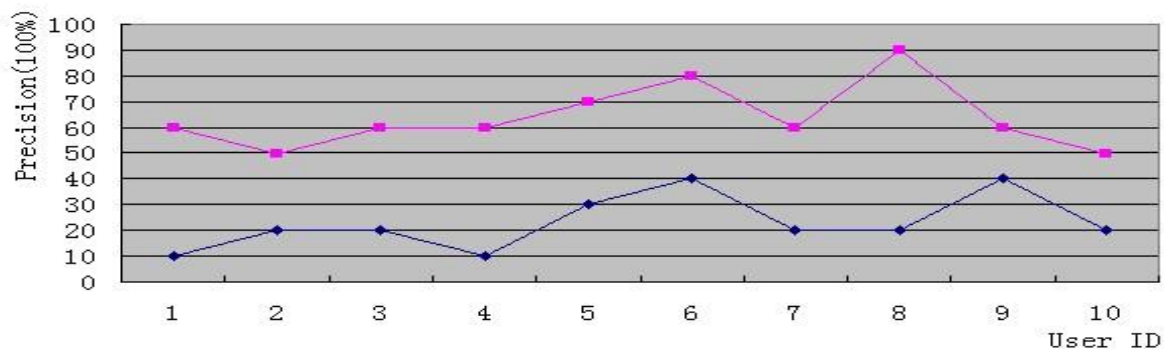

Figure 4. Precision Comparison between Improved and Traditional System

As shown in Figure 4, the accuracy of the first way without the location context is between $10 \%$ 40\%. But the improved system in this paper can raise the accuracy to $60 \% \sim 90 \%$, the average accuracy is about $70 \%$. The test result means the improved system greatly increases the accuracy of service discovery.

The algorithm in this paper has been tested in the above 2 mentioned experiments. Although it is more time-consuming, still acceptable for the users and the accuracy and efficiency has been improved significantly.

\section{Conclusion and Future Work}

Service discovery is the foundation of realizing the service recommendation to support mobile service personalization. This paper proposes a strategy of service discovery.

In this paper, it solves the problem of the weight distribution for user preference and location context in the services discovery. Our research work consists of several aspects. First of all, it discusses the modeling of LCAMSP which is based on the traditional methods. Then, it describes the user information on the semantic level accurately. Then, it makes a systematic analysis on user preference and proposed a method which can dynamically calculate the weight for various attributes. Furthermore, it combines the user preference and location context to conclude the final recommendation results. Finally, it designs the experiments to test the feasibility. The experiment result supports the mobile service discovery based on location context better, and it also shows that the precision of service discovery is improved.

Preference characteristics and the changes of the location context play a different role in the development of service discovery strategy to meet the personal needs of user. More and more researchers are concerned about this research aspect of mobile Internet. In recent years, LBS becomes a hot spot with the development of the masses of services, it provides more opportunities for the future research. In the future, we will continue our research to study the perfection mechanism of the self-adjust mode for locationbased service personalization under the mobile Internet environment. 


\section{Acknowledgments}

Our research is supported by National Natural Science Foundation of China (Project Number.61074135) and Shanghai Leading Academic Discipline Project (Project Number.J50103). We should show our great thanks to all of our hard working fellows in the above projects.

\section{References}

[1] J. Zhao, C. Zheng and D. Zhou, "Design and implementation of a Location-based service platform", International Conference on Advanced Communication Technology, vol. 1, (2008), pp. 529-533.

[2] G. Retscher, "Location determination in indoor environments for pedestrian navigation", Position and navigation symposium, IEEE, (2006), pp. 547-555.

[3] E. Bertino, E. Ferrari and A. Perego, "A general framework for web content filtering", World Wide Web, vol. 13, (2010), pp. 215-249.

[4] E. Jembere, M. O. Adijun and S. S. Xulu, "Ming Context-based User preferences for m-Services Applications", International Conference on Web Intelligence, (2007).

[5] E. Maximilien and M. Singh, "A framework and ontology for dynamic Web services selection", IEEE Internet Computing, vol. 8, no. 5, (2004) September-October, pp. 84-93.

[6] L. Hu, K. Zhao, S. Ying and R. Chen, "A semantic web service description language", International Conference on Information Engineering, vol. 2, (2009), pp. 449-452.

[7] H. Liu, C. Shen, D. Yang and L. Liu, "The summary of basic technology research for semantic web services", Journal of Jilin University(Information Science), (2010), pp. 47-54.

[8] Y. Yang, L. Chen, B. Xie and L. Pan, "The research of services discovery based on user context Clustering".

[9] W. Li, "The research of context discovery and anomaly detection for the mobile Internet with location".

[10] H. Xu, X. Wu, X. Li and B. Yan, "The research and comparison of Internet recommendation system", Journal of Software, (2009), pp. 350-362.

[11] H. Cai, "The service mode of personalized information recommendation", Journal of Information, (2006), pp. 116-121.

[12] L. Wu and L. Liu, "The summary of user modeling in personalized recommendation system", Journal of Intelligence.

[13] Z. Xu and L. Shi, "Computer Application and Software", The research of service discovery algorithm based on semanteme, (2006).

[14] K. Li and L. Jiang, "The research of semantic web service discovery algorithm based on constraint extraction and structure analysis", Computer Engineering \& Science, (2013).

\section{Authors}

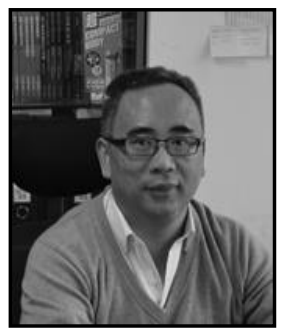

Mingjun Xin is a professor at School of Computer Engineering and Science, Shanghai University. He received his PH.D. from School of Computer Science and Technology, North western Polytechnical University, Xi'an Chinain 2001. His research interests are Quality of Service (QoS), Location-based Services, mobile platform, service recommendation and software engineering.

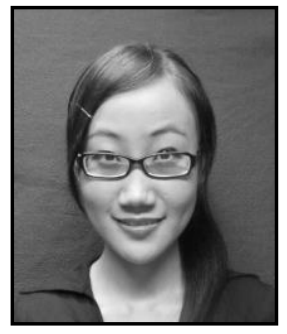

Liyuan Zhou was born in 1990, studying the M.S. degree in School of Computer Science and Engineering from Shanghai University. Her research interests include recommendation system, machine learning etc. 


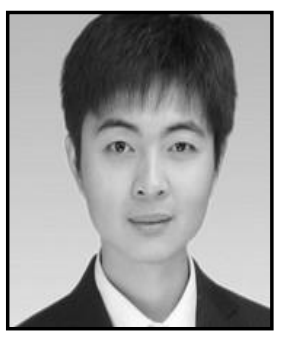

Han Cao was born in 1989. He received his M.S. degree from School of Computer Science and Engineering from Shanghai University. Currently, he is working in China Merchants Bank.

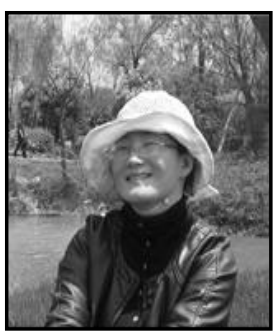

Niu Zhihua was born in 1976. She received her Ph.D degree at Xidian University. Now she is a lecturer at the School of Computer Engineering and Science, Shanghai University. Her main research fields ar cryptography and information security. 
International Journal of Grid Distribution Computing Vol.7, No.6 (2014) 